\title{
Weld Flaw Detection Based on Likelihood Estimation and Wavelet Transform
}

\author{
Mohanasundari L, Sivakumar P
}

\begin{abstract}
The metals are fused at high temperature using various welding methods to form mechanical structures. The structural stability is achieved in metals by the proper welding process utilized. The failure may result in disaster and huge investment has to be incurred towards building the flawless structures. It is always preferred to check the quality of the weld before the final welded structure is used for its actual application. Though visual inspections could solve problems tentatively valid for low production rates, there are scenarios where visual inspection fails and needs high end methods to analyze the quality of welded joints. Several measurement techniques have evolved and help the user community. The objective of this paper is reviewing such earlier methods in the relevant domain of research and tabulates the various merits and demerits so as to find a method to overcome earlier drawbacks. Further, edge detection is done based on adaptive thresholding method to find the weld flaws.
\end{abstract}

Index terms: Radiographic image, classification, segmentation, enhancement, genetic algorithm.

\section{INTRODUCTION}

Out of all industrial processes existing in world, most significant and ranks high is the process of welding. Any further operations on the welded part in industries or direct usage of such finished product by consumer is depended on such welded structures. Welding is done in many ways which essentially needs a high temperature to be produced in the order of melting point of the metals to be joined. Moreover, welding is an art of mechanical process which may be of indoor or outdoor in nature. The performance of welding processes depends on the welding methods, environmental conditions and the accuracy in spotting the area to be welded.

\subsection{Basic Methodology Of Welding}

The sourceof the test images would be obtained from any type of imaging methods such as ultrasonic imaging, X-ray imaging or a direct positive imaging method. However, the defect identification depends on the efficient classification performed on the test images. The basics of imaging techniques rely on transmitting the energy in the form of ultrasonic waves, X-ray or Infrared on the metal joints and acquiring the reflected energy from the metal surface. A classification method is used to distinguish the feature sets of counter bore, counter bore and echoes and waveforms with flaws. The k-nearest neighbor algorithm is used for classifying the waveform features.

\footnotetext{
Revised Manuscript Received on 14 August, 2019.

Mohanasundari L, Assistant professor, Department of Electronics and Communication Engineering, Kingston Engineering College, Vellore, Tamilnadu, India (Email: 1msecerec@gmail.com)

Sivakumar P, Professor, Department of Electronics and Communication Engineering, Dr. N.G.P. Institute Of Technology, Coimbatore, Tamilnadu, India(Email: sivakumar.poruran@gmail.com)
}

As a next stage, the three different classifiers such as Gaussian probability density function classifier, Fisher linear discriminant classifier and F-nearest neighbor classifier were trained. Feature sets were extracted and applied to classifiers could result accuracy in the range of $92-97 \%$. But the same method failed when tested with a different database and could not classify anywhere between $79-88 \%$. It is fair to note that neural network had been used to construct a relationship between weld quality parameters and weld pool geometry in TIG (Tungsten Inert Gas) TIG welding. Simulated annealing based optimization is performed to identify the optimal welding parameters which satisfy the required quality metrics. Fuzzy clustering method is used to classify the aluminium weld quality and to obtain the required. In order to ensure a quality welding in the areas of aerospace, automotive sectors and nuclear plant constructions, arc welding and laser welding had been introduced in the scenario by the research community. Such arc weld tests conducted on steel are reported while using temperature profile obtained from plasma spectra used to extract feature vectors using principal component analysis. Sequential classification using NN could prove a better correlation between the ANN outputs and temperature profiles.

\section{LITERATURE REVIEW}

This critical survey is intended to provide a wide review on existing defect detection methods and to find out the problems and accuracy measures obtained in those papers. The survey has been conducted by focusing the problem only towards the classification issues irrespective of the type of source image used for analysis. Basic principle behind the manual defect detection is by manual inspection. Unfortunately micro sized defects and defects which are beyond the direction vision are not observable and hence it needs intelligent methods to find it. Also, such methods lead to automation in testing large data base in shorter duration.

2.1 "An Imaging and Measurement System for Robust Reconstruction of Weld Pool during Arc Welding" - Zhen Zhou Wang (2015)

The proposed method makes use of the strong penetrability feature of the laser to evade the intervention of arc light and to obtain the weld pool surface information. The surface is reconstructed by one-shot structured light projection. To increase the robustness of the measurement system, least deformation principle is proposed to rectify the coefficient errors of the computed plane equation.

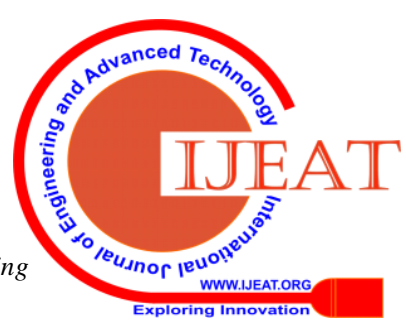




\section{WELD FLAW DETECTION BASED ON LIKELIHOOD ESTIMATION AND WAVELET TRANSFORM}

2.2 "A New Approach For Segmentation And Quantification Of Cells Or Nanoparticles" - Zhen Zhou Wang (2016)

The proposed morphological erosion method calculates the optimal threshold and segments or quantifies the cells of nanoparticles automatically and efficiently whereas the Threshold selection method calculates the optimal threshold using slope difference of the smoothed histogram. The proposed method has the prospective to promote many automated microscopic imaging applications.

2.3 “Robust Welding Seam Tracking And Recognition" Xianghui Li And Xinde Li (2017)

To reduce the impact of interference and to obtain the center smoother line of welding seam, the sequence gravity method (SGM) is used. To improve the accuracy and realtime performance, the double-threshold recursive least square method is used so that it fits the curve obtained by SGM. It is a time consuming process.

\section{LIKELIHOOD ESTIMATION METHOD}

Likelihood estimation is an iterative algorithm to group the input pixels into two categories based on calculating the expected value and maximizing the expected value with the help of updating the weight. This is done individually for all the pixels in the image. The expected value is calculated for every pixels in an image with assumed weight value and given to maximization step. The maximization step will update the weight value and given to the expectation step, this process continuous in an iterative manner until the expected weight value and updated weight value remains the same. Image acquisition, Preprocessing, Feature extraction (spatial feature), Classification based on training and testing, Performance Evaluation are the process of weld flaw detection. The process flow is used for detecting the interior weld flaw in an input image and gives the final segmented image detected by the algorithm.

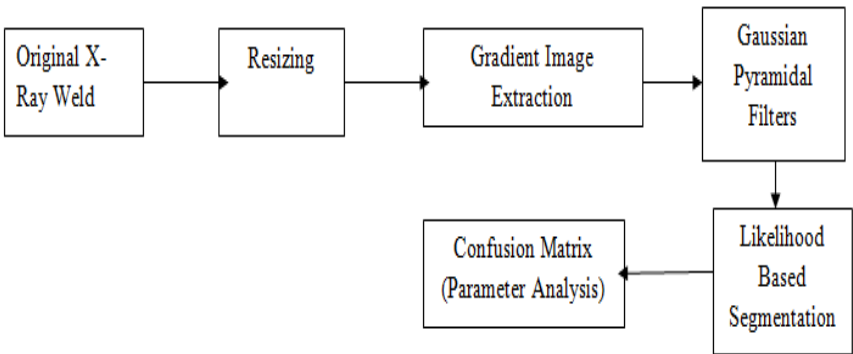

Figure 3.1 Block Diagram of Likelihood Estimation

\subsection{Original X-Ray Weld Image}

The input image is obtained through $\mathrm{X}$-ray welding process where the large set of such input images are taken from GRIMA standard x-ray database.

\subsection{Resizing}

The resizing standard is generally $256 \times 1024$ (rows $\times$ columns), the given original input image is resized to the standard range to analyze every input database with standard image size.

\subsection{Gradient Image Extraction}

The gradient image extraction is the rate of change of pixel values (abrupt change) used for edge detection.
The gradient image formation is given by,

$g x=i(x+n, y)-i(x-n, y)$

$g y=i(x, y+n)-i(x, y-n)$

The gradient pixel value is given by,

$\mathrm{g}=\sqrt{\mathrm{gx}^{\wedge}} 2+\mathrm{gy}^{\wedge} 2$

The above formula is used to obtain the gradient pixel value in an image to detect the weld flaw edges and the detected edge image is given to gaussian pyramidal filter for further processing.

\subsection{Guassian Pyramidal Filter}

In image processing, gaussian filter is widely used to reduce the effects on image noise and reduce detail. The gaussian pyramidal filter is used to fine tune the edges. The pyramidal filter gives two levels of decomposition to obtain final level output by eliminating the spurious pixels (false) by blurring it and gives final resolution output.

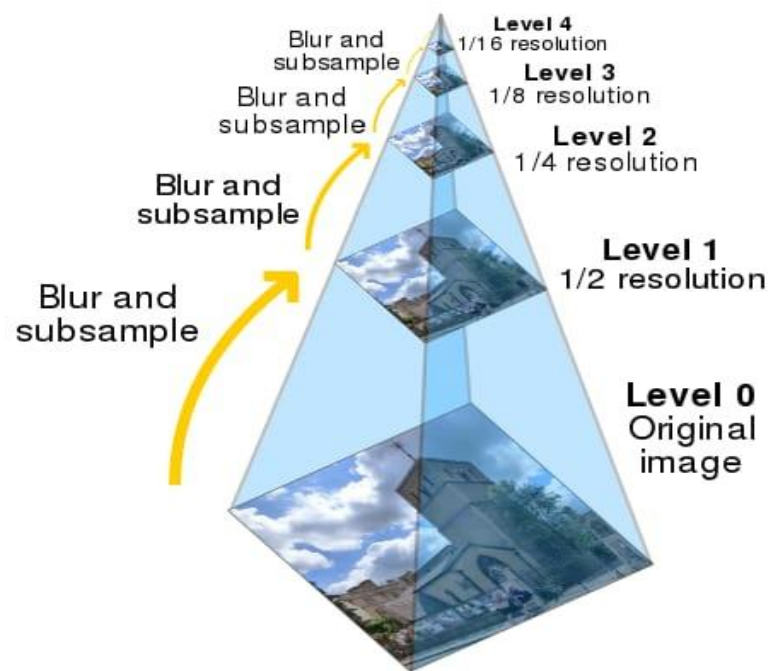

Figure 3.2 Gaussian Pyramidal Resolution Image.

The gaussian pyramidal filter has many level of decomposition as per the requirement, with the help of gaussian filter, the image with better resolution is obtained and accurate detection of weld pixels is done by eliminating the unwanted pixels. The image result from gaussian pyramidal filter is given to the likelihood estimation algorithm method which segments an image into two groups, one group contains the flaw pixel and other group contains the pixel without flaw.

\subsection{Likelihood Based Segmentation}

The EM (EXPECTATION MAXIMIZATION) algorithm is known as likelihood estimation algorithm. It is an iterative algorithm where for each pixels in an image the expected value is calculated and given for maximization step. The updated weight value is substituted in expectation step and this process is done in an iterative manner until the probability of weight value remains same. The procedure in an expectation maximization algorithm closely follows the K-means clustering algorithm. The accuracy of EM algorithm depends on the initial cluster values.. 


\subsection{Confusion Matrix}

A confusion matrix (or error matrix) is a table showing the relation between reference image and classification result. The performance metrics such as sensitivity, specificity and accuracy are analyzed using this error matrix. The performance metric parameters are given below.

\subsection{1 sensitivity}

Sensitivity is a measure used to analyze how the proposed system correctly classifies weld flaw pixels efficiently. The sensitivity is measured as follows:

Sensitivity $=(\mathrm{TP}) /(\mathrm{TP}+\mathrm{FN})$

\subsubsection{Specificity}

Specificity is a measure to find how the proposed system correctly identifies the negative classifiers during the detection of weld flaw pixels which is measured as follows:

Specificity $=(\mathrm{TN}) /(\mathrm{TN}+\mathrm{FP})$

\subsubsection{Accuracy}

Accuracy is a statistical measure to analyze the weld flaws in an optimized way. Among the various cases examined, both true positives and true negatives are calculated. The accuracy value is calculated as follows:

Accuracy $=(\mathrm{TP}+\mathrm{TN}) /(\mathrm{TP}+\mathrm{FP}+\mathrm{FN}+\mathrm{TN})$

These three performance metric value analyzed by the confusion matrix decides whether the choosed algorithm correctly detects the weld flaw present in an given input image with better accuracy.The drawback is that the spatial domain provides less accuracy. To overcome this, the Frequency domain method is used to obtain better accuracy using "WAVELET TRANSFORM" method.

\section{WAVELET TRANSFORM METHOD}

The concept of wavelet transform is that an input image is obtained from GRIMA database and it is divided into two sub bands, low frequency band and high frequency band. The low frequency band is divided into many number of sub bands since it has got more information than the higher frequency bands. This information is used to calculate the two parameters (1) energy (2) entropy. Image acquisition and Preprocessing, Feature extraction (frequency domain), SVM Classification based on training and testing, Performance evaluation are the process of weld flaw detection. The process flow is used for detecting the weld flaw in an image using wavelet transform method. The energy and the entropy are two measuring parameters. The energy measures the pixel pair repetition and the entropy extracts information in each sub-band of image. The SVMRBF used energy and entropy value to segment an image into four decomposition level to obtain better accuracy

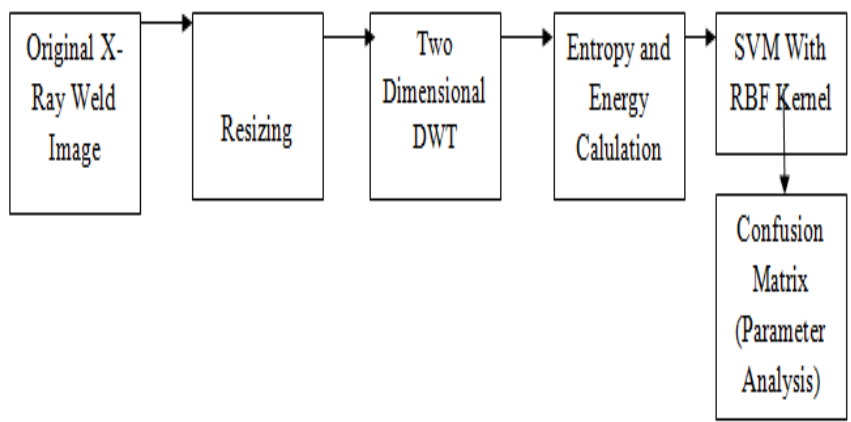

\subsection{Two Dimensional Dwt}

The Discrete wavelet transform is used in signal analysis since it is used to obtain both the location and frequency information. A decrease in values of local energy and entropy is also an indicator of flaw. In addition, scene becomes bright when there is flaw and this leads to an increase in intensity in the particular flaw regions. All of these clues are combined to reach a final decision of finding the flaw. It uses mainly two parameters such as energy and entropy to detect the flaw at each sub-band. The value will be compared to threshold to obtain the grouping of pixels depending on flaw and without flaw.

\section{Energy}

Energy (E) is an extent of pixel pair repetitions that measures the uniformity of an image respectively. The value of the energy is more when the pixels are similar to one another. The wavelet based energy feature is defined as

$$
E(I)=\sqrt{\sum_{i=0}^{N-1} \sum_{j=0}^{N-1} M_{i, j}}
$$

Where, E (I) is the energy, Mi, $\mathrm{j}$ be the sub band coefficients at location $\mathrm{i}, \mathrm{j}$ and $\mathrm{N}$ be Sub bands.

\section{Entropy}

Entropy is defined as the statistical measurement of the uncertainty degree and the information content of a message exists in a system. The entropy gives a measurement of information and therefore it is adapted to characterize an image each of which is considered as a specific message.

Entropy of an image is stated as a quantity of an image, in which the information amounts of each sub-band are is coded by a compression algorithm. The wavelet based entropy feature is defined as

$$
\text { Entropy }=-\sum_{j=1}^{n} P\left(x_{j}\right) \log _{2} P\left(x_{j}\right)
$$

\subsection{SvmWithRbf Kernel}

The general aim of analyzing the pattern is to study the various relations like clusters, principal components, classification ) in datasets. Using the SVM classification technique, the input test vectors are identified. In this supervised learning algorithm,the training vectors are used to train the system. It is desirable to generate maximum distance between the training sets. So that better accurate classification can be obtained. In SVM classification, the inner product is converted into various forms of kernel functions

$$
\mathrm{k}\left(\mathrm{x}_{\mathrm{i}}, \mathrm{x}_{\mathrm{j}}\right)=\exp \left(-\gamma\left\|\mathrm{x}_{\mathrm{i}}-\mathrm{x}_{\mathrm{j}}\right\|^{2}\right)(\text { RBF kernel })
$$

In radial basis function, the norm is usually Euclidean distance, although other distance are also possible. 


\subsection{Performance Parameter Metrics}

The validation metrics are used to calculate the performance parameter metrics. The validation metrics are TP, TN, FP and FN. The input image and ground truth image are compared and ground truth image is considered as base image. It contains foreground image denotes by 'white pixels' and background image denotes by 'black pixels'. In True positive and True negative pixels are segmented as foreground and background respectively. In True negative and false positive pixels are segmented as background and foreground respectively.

Table 6.2 Parameter Values of Wavelet Transform

\begin{tabular}{|c|c|c|c|c|}
\hline IMAGES & LEVELS & ACCURACY & SENSITIVITY & SPECIFICITY \\
\hline \multirow{4}{*}{1} & LEVEL1 & 0.916 & 0.91 & 0.833 \\
\hline & LEVEL2 & 0.908 & 0.883 & 0.933 \\
\hline & LEVEL3 & 0.997 & 0.91 & 0.91 \\
\hline & LEVEL4 & 0.958 & 0.916 & 0.9 \\
\hline \multirow{4}{*}{2} & LEVEL1 & 0.916 & 0.91 & 0.833 \\
\hline & LEVEL2 & 0.908 & 0.866 & 0.95 \\
\hline & LEVEL3 & 0.997 & 0.91 & 0.91 \\
\hline & LEVEL4 & 0.966 & 0.933 & 0.93 \\
\hline \multirow{4}{*}{3} & LEVEL1 & 0.916 & 0.91 & 0.833 \\
\hline & LEVEL2 & 0.908 & 0.866 & 0.95 \\
\hline & LEVEL3 & 0.997 & 0.91 & 0.95 \\
\hline & LEVEL4 & 0.958 & 0.916 & 0.96 \\
\hline \multirow{4}{*}{4} & LEVEL1 & 0.908 & 0.91 & 0.816 \\
\hline & LEVEL2 & 0.908 & 0.866 & 0.95 \\
\hline & LEVEL3 & 0.997 & 0.91 & 0.96 \\
\hline & LEVEL4 & 0.941 & 0.883 & 0.96 \\
\hline \multirow{4}{*}{5} & LEVEL1 & 0.916 & 0.91 & 0.833 \\
\hline & LEVEL2 & 0.9 & 0.866 & 0.933 \\
\hline & LEVEL3 & 0.997 & 0.91 & 0.95 \\
\hline & LEVEL4 & 0.95 & 0.9 & 0.6 \\
\hline
\end{tabular}

The above Table 6.1 and 6.2 shows the performance metrics of likelihood estimation method and wavelet transform method

\section{CONCLUSION}

The basic principle behind the likelihood estimation based segmentation method and wavelet transform method is to detect the weld flaw in $\mathrm{x}$-ray weld images. The performance metrics were calculated. The accuracy obtained by wavelet transform (frequency domain) is found to be more at decomposition level 3 , when compared to likelihood estimation method.

V. RESULTS

Table 6.1 Parameter Values of Likelihood Estimation

\begin{tabular}{|l|l|l|l|}
\hline $\begin{array}{l}\text { IMAGE } \\
\text { S }\end{array}$ & $\begin{array}{l}\text { ACCURAC } \\
\text { Y }\end{array}$ & $\begin{array}{l}\text { SENSITIVIT } \\
\text { YPECIFICIT }\end{array}$ & \begin{tabular}{l} 
Y \\
\hline 1
\end{tabular} \\
\hline 2 & 0.8974 & 0.9374 & 0.3951 \\
\hline 3 & 0.9943 & 0.9984 & 0.3391 \\
\hline 4 & 0.9960 & 0.9965 & 0.5526 \\
\hline 5 & 0.9896 & 0.9900 & 0.8304 \\
\hline
\end{tabular}




\section{REFERENCES}

1. Li XH, Li XD, Khyam MO, Ge SZS, "Robust welding seam tracking and recognition," IEEE Sensors Journal, vol. 17 , no. 17, pp. 5609-5617, 2017.

2. Gstalter M, Chabrol G, Bahouka A, Serreau L, Heitz JL, Taupier G, Dorkenoo KD, Rehspringer JL, LeclerS,"Stress-induced birefringence control in femtosecond laser glass welding," Applied Physics a Materials Science \& Processing, vol. 123, no. 11, pp. 714(1-6), 2017.

3. Wanjara P, Monsarrat B, Larose S, "Gap tolerance allowance and robotic operational window for friction stir butt welding of AA6061," Journal of Materials Processing Technology, vol. 123, no. 11, pp. 631-640, 2013.

4. Wilhelm G, Gott G, Uhrlandt D, "Study of flux-cored arc welding processes for mild steel hardfacing by applying high-speed imaging and a semi-empirical approach," Welding in the World, vol. 61, no. 5, pp. 901-913, 2017.

5. Na Lv, YanlingXu, Sichen Li, Xinwen Yu, Shanben Chen, "Automated control of welding penetration based on audio sensing technology," Journal of Materials Processing Technology, vol. 250, pp. 81-98, 2017.

6. Rodriguez-Martin M, Rodriguez-Gonzalvez P, GonzalezAguilera D, Fernandez-Hernandez J, "Feasibility study of a structured light system applied to welding inspection based on articulated coordinate measure machine Data," IEEE Sensors Journal, vol. 17, no. 13, pp. 4217-4224, 2017.

7. G. Saeed, M.J. Lou and Y.M. Zhang,"Computation of 3D weld pool surface from the slope field and point tracking of laser beams", Measurement Science and Technology, Vol. 15, No. 2, pp.389-403, 2004.

8. Z.Z. Wang, "An imaging and measurement system for robust reconstruction of weld pool during arc welding", IEEE Trans. Ind. Electron. Vol. 62, No. 8, pp. 51095118, 2015 [9] H. S. Song and Y.M. Zhang, "Measurement and analysis of three-dimensional specular gas tungsten arc weld pool surface", Welding Journal, Vol. 87, pp. 85-95, 2008. [10] Y.K. Liu and Y.M. Zhang, "Iterative local ANFIS-based human welder intelligence modeling and control in pipe GTAW process: a data-driven apporach", IEEE/ASME Trans. Mech. Vol. 20, No. 3, pp. 1079-1088, 2015.

9. Y. Q. Wei, N. S. Liu, X. Hu, and X. Ai, "Phasecorrection algorithm of deformed grating images in the depth measurement of weld pool surface in gas tungsten arc welding," Optical Engineering, vol. Vol. 50, No. 5, pp. 057209-057209-6,2011.

10. Y.M. Zhang, E. Liguo and R. Kovacevic, "Active metal transfer control by monitoring excited droplet oscillation", Welding J. Vol. 77, No. 9, pp. 388S-395S, 1998.

11. I. Lertrusdachakul, A. Mathieu and O. Aubreton, "Vision-based control of wire extension in GMA welding", Int J AdvManufTechnol, Vol. 78, No. 5, pp. 1201-1210, 2015.

12. Z.Z. Wang, "A laser back-lighting based metal transfer monitoring system for robotic gas metal arc welding", Robotics and Computer-Integrated Manufacturing, Vol 38, Issue. C, pp. 52-66, 2016 [15] G.J. Zhang, Z.H. Yan and L. Lin, "Reconstructing a three-dimensional PGMAW weld pool shape from a two-dimensional visual image", MeasSciTechnol, Vol. 17, No. 7, pp. 1877-1882, 2006 13. X. J. Ma and Y.M. Zhang, "Gas tungsten arc weld pool surface imaging: modleing and processing", Welding Journal, Vol. 90, pp. 85-94, 2011.

13. Y. Shi, G. Zhang, X.J. Ma, Y.F. Gu, J.K. Huang and D. Fan, "Laser-vision-based measurement and analysis of oscillation frequency in GMAW-P", Welding Journal, Vol. 94, pp. 176-187, 2015.
14. Z.Z. Wang, "Monitoring of GMAW weld pool from the reflected laser lines for real time control", IEEE Trans. Ind. Infrom. Vol. 10, No. 4, pp. 2073-2083, 2014 16.R. M. Haralick, K. Shanmugam, I. Dinstein, "Textural Features for Image Classification".IEEE Transactions on Systems, Man, and Cybernetics. SMC-3 (6): 610-621, 1973.

15. Mohanasundari L, Sivakumar P, Chitra N, " Feature Extraction through Chaotic Metrics for Weld Flaw Classification ", International Journal of Innovative Technology and Exploring Engineering (IJITEE) ISSN: 2278-3075, Volume-8 Issue-4S, February 2019 